\title{
The fabrication of small molecule organic light-emitting diode pixels by laser-induced forward transfer
}

\author{
J. R. H. Shaw-Stewart, ${ }^{1,2, a)}$ T. Mattle, ${ }^{2}$ T. K. Lippert, ${ }^{2, b)}$ M. Nagel, ${ }^{1}$ F. A. Nüesch, ${ }^{1,3, c)}$ \\ and A. Wokaun ${ }^{2}$ \\ ${ }^{1}$ Laboratory for Functional Polymers, Empa Swiss Federal Laboratories for Materials Science and \\ Technology, Überlandstrasse 129, CH-8600 Dübendorf, Switzerland \\ ${ }^{2}$ Materials Group, General Energy Research Department, Paul Scherrer Institut, CH-5232 Villigen-PSI, \\ Switzerland \\ ${ }^{3}$ Section de science et génie des matériaux, EPFL, CH-1015 Lausanne, Switzerland
}

(Received 24 August 2012; accepted 4 January 2013; published online 23 January 2013)

\begin{abstract}
Laser-induced forward transfer (LIFT) is a versatile organic light-emitting diode (OLED) pixel deposition process, but has hitherto been applied exclusively to polymeric materials. Here, a modified LIFT process has been used to fabricate small molecule $\mathrm{Alq}_{3}$ organic light-emitting diodes (SMOLEDs). Small molecule thin films are considerably more mechanically brittle than polymeric thin films, which posed significant challenges for LIFT of these materials. The LIFT process presented here uses a polymeric dynamic release layer, a reduced environmental pressure, and a well-defined receiver-donor gap. The $\mathrm{Alq}_{3}$ pixels demonstrate good morphology and functionality, even when compared to conventionally fabricated OLEDs. The $\mathrm{Alq}_{3}$ SMOLED pixel performances show a significant amount of fluence dependence, not observed with polymerical OLED pixels made in previous studies. A layer of tetrabutyl ammonium hydroxide has been deposited on top of the aluminium cathode, as part of the donor substrate, to improve electron injection to the $\mathrm{Alq}_{3}$, by over $600 \%$. These results demonstrate that this variant of LIFT is applicable for the deposition of functional small molecule OLEDs as well as polymeric OLEDs. (C) 2013 American Institute of Physics. [http://dx.doi.org/10.1063/1.4788710]
\end{abstract}

\section{INTRODUCTION}

The application of organic light-emitting diodes (OLEDs) in electronic displays has led to a large increase in research into OLED deposition and patterning techniques. Laserinduced forward transfer (LIFT) has been developed to enable fine patterned deposition of solid thin-films, such as those used in OLEDs. Additional advantages of laser-processing include high-throughput volumes, meaning the LIFT process is suited to large-scale applications. LIFT also transfers solid layers intact, meaning that layers on the donor substrates can be formed from processes that would be otherwise incompatible with other layers in the device. A final advantage is that the use of an intermediate polymer layer (dynamic release layer (DRL)) to absorb the laser light allows for the transfer of materials that would otherwise be sensitive to the laser radiation.

Whilst "direct-write" laser deposition techniques have been investigated for a number of years, ${ }^{1-3}$ scientific research into the process has really taken off in the past few years. In the field of OLEDs, this research is particularly important given that active-matrix OLED displays are already widely available on the market. ${ }^{4}$ LIFT is a term that encompasses a range of laser deposition techniques; the variant used in this article is summed up in Fig. 1, and an overview

\footnotetext{
${ }^{a)}$ Current address: Grant Institute, University of Edinburgh, The King's Buildings, Edinburgh EH9 3JW, United Kingdom. Electronic mail: james. shaw-stewart@ed.ac.uk.

b)Electronic mail: thomas.lippert@psi.ch.

${ }^{c)}$ Electronic mail: frank.nueesch@empa.ch.
}

of the different LIFT technique classifications is shown in a previous article. $^{3}$ Our variant of LIFT is unique in that it uses a thin sacrificial DRL of triazene polymer (TP) to propel the target layers across intact. OLEDs have been fabricated using our variant of LIFT, ${ }^{5,6}$ as well as another LIFT variant where the layer was not transferred intact, blister-actuated LIFT (BA-LIFT) ${ }^{7}$ and a commercially developed technique termed laser induced thermal imaging (LITI). ${ }^{8}$ The technique shown here, in Fig. 1, has previously only been applied successfully to polymeric light-emitting layers. With recent improvements to the technique, ${ }^{9,10}$ new approaches have been tried, such as transferring different types of pixels sideby-side $^{11}$ and sequentially transferring the light-emitting layer and the cathode in separate steps. ${ }^{12}$

Here, we present the first transfers of small molecule OLED pixels using any form of LIFT (hereafter called LIFTed pixels, as opposed to conventionally fabricated devices), where the layers are transferred intact across a gap. $\mathrm{Alq}_{3}$ has been deposited using BA-LIFT, ${ }^{9}$ and smallmolecule OLEDs using other materials were fabricated, but the organic layer was transferred as a powder, and the cathode was subsequently evaporated after the laser deposition process. ${ }^{7}$ Our group has also investigated $\mathrm{Alq}_{3}$ LIFT before, during the optimisation of the process, ${ }^{10}$ but functional pixels were not successfully fabricated.

$\mathrm{Alq}_{3}$ was the first material used for low voltage, efficient OLED devices by Tang and Van Slyke in $1987 .{ }^{13}$ It is a small molecule material, in contrast to the polymeric materials used as the light-emitting layers in previous LIFT studies (see references for information on the polymers): $\mathrm{MEH}-$ 


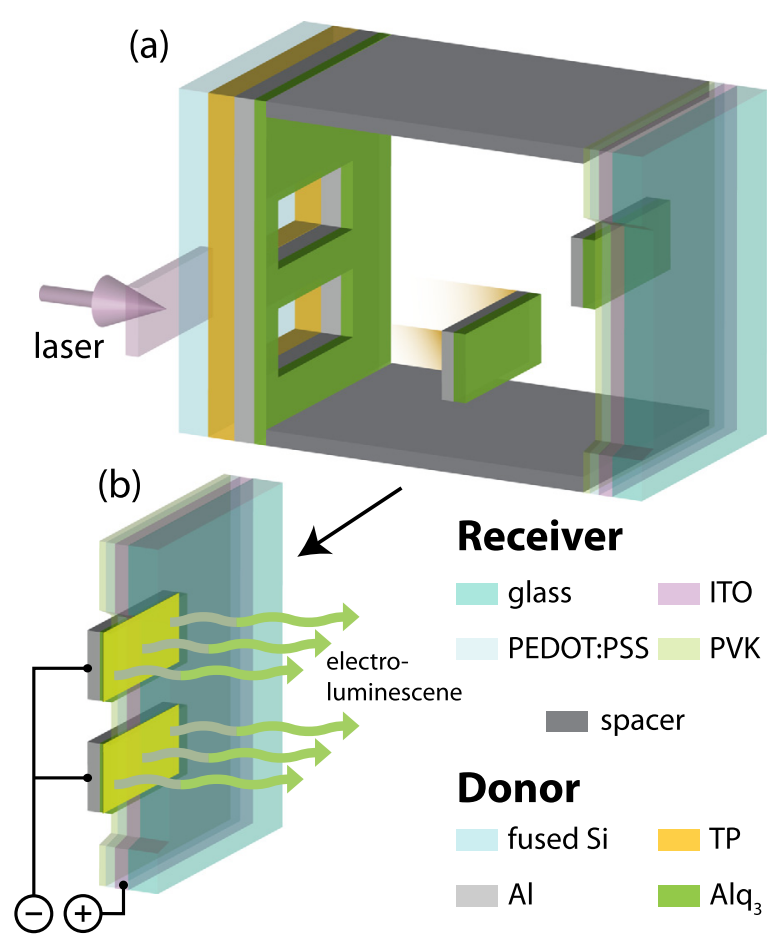

FIG. 1. A scheme showing the LIFT process. The laser beam punching out an $\mathrm{Alq}_{3}$ pixel for transfer from the donor to the receiver substrate is shown in (a), and pair of electroluminescent pixels are shown with a bias applied in (b).

PPV,${ }^{5,6}$ PFO,${ }^{11}$ and PFN. ${ }^{12}$ Because of the lower mechanical and thermal stabilities of small-molecule organic films relative to polymers, the functionality of these devices is of particular interest.

An improvement to the LIFTed $\mathrm{Alq}_{3}$ pixel performance has been demonstrated, in this article, with the use of tetrabutyl ammonium hydroxide (TBA), see Fig. 2. TBA was first proposed as a chemical base for the fabrication of a monolayer on indium-doped tin oxide (ITO), and was shown to reduce the work function of ITO significantly. ${ }^{14}$ At the time, it was not possible to test TBA as an electron injecting layer on the cathode because inverse architectures were not widespread, but it was used to block hole injection. ${ }^{15}$ Here, we use TBA on the cathode because the fabrication of the donor substrates allows for direct modification of the Al before the $\mathrm{Alq}_{3}$ is deposited (see Sec. II A).

\section{EXPERIMENTAL}

\section{A. Substrate preparation}

The donor substrates use UV-transparent fused silica slides $(25 \mathrm{~mm} \times 25 \mathrm{~mm} \times 1 \mathrm{~mm})$, cleaned thoroughly with

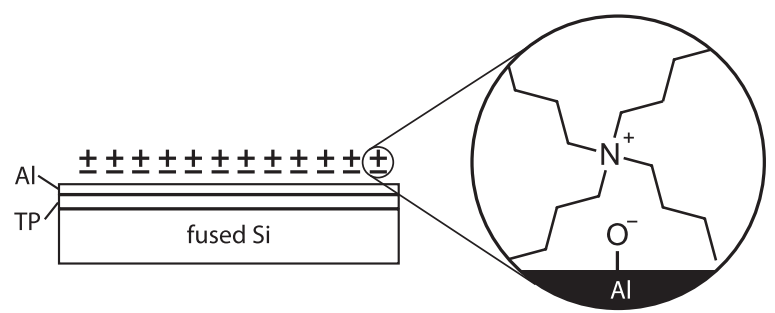

FIG. 2. The chemical structure of TBA, and its location on the donor substrate: a monolayer on top of the aluminium. Based on a figure from an article by Nüesch et al. ${ }^{15}$ solvent and detergent baths, and UV-ozone treatment. The fused silica substrates are spin-coated at $1500 \mathrm{rpm}$ with $3 \mathrm{wt}$. \% solutions of TP, first synthesised in $1993 .{ }^{16}$ Profilometry shows that this gives a thin film thickness of $190 \mathrm{~nm} \pm 10 \mathrm{~nm}$. The cathode was made by thermally evaporating $90 \mathrm{~nm}$ films of aluminium onto the TP films at pressures below $10^{-5}$ mbar, with the thickness measured using a quartz-crystal microbalance. Finally, $80 \mathrm{~nm}$ aluminium tri-8-hyroxyquinoline $\left(\mathrm{Alq}_{3}\right.$, sublimed grade, Sigma Aldrich) was deposited under the same conditions, on top of the Al. For the addition of TBA onto the $\mathrm{Al}$, the substrates were removed from the vacuum chamber and a $10^{-4} \mathrm{M}$ TBA in methanol solution was spin-coated at $1500 \mathrm{rpm}$ before the substrate was placed back into the vacuum chamber for the $\mathrm{Alq}_{3}$ deposition.

The receiver substrates use pre-patterned $140 \mathrm{~nm}$ thick ITO glass slides $(25.4 \mathrm{~mm} \times 25.4 \mathrm{~mm} \times 1 \mathrm{~mm})$. These were spin-coated with two hole-transporting layers (HTLs): $60 \mathrm{~nm}$ poly[3,4-ethylene dioxythiophene] blended with poly[styrene sulfonate] (PEDOT:PSS) (Clevios P Al4083) and $40 \mathrm{~nm}$ of poly[N-vinylcarbazole] (PVK, Aldrich, $\left.\bar{M}_{w}=1100000\right)$.

The receiver substrates were also used as the base for conventionally fabricated devices, where the $\mathrm{Alq}_{3}$ was evaporated using the same conditions as above, but directly onto the PVK. Al was then evaporated through a shadow mask which defined the shape ( 7 and $3.5 \mathrm{~mm}^{2}$ circles) of the resultant devices with the architecture: ITO/PEDOT:PSS/PVK/ $\mathrm{Alq}_{3} / \mathrm{Al}$.

\section{B. LIFT setup}

The samples were kept in an inert nitrogen environment throughout the preparation process, and only removed briefly to be placed into, and removed from, the LIFT vacuum chamber. The receiver substrate was placed opposite the donor substrate with a $10 \mu \mathrm{m}$ steel spacer between which gave a gap width of $15 \mu \mathrm{m}$ from interferometry. ${ }^{10}$ Both the donor and receiver substrates are shown with the spacer in Fig. 1(a). The samples were placed in a vacuum chamber where a dry rough pump reduces the pressure to 1 mbar. Single pulses of a XeCl excimer laser $(\lambda=308 \mathrm{~nm}, \tau=30 \mathrm{~ns})$ were used for the ablation of the TP DRL.

The LIFT procedure including the details of the laser setup and optimization of the gap width and environmental pressure for the LIFT process are explained in detail elsewhere. ${ }^{10}$ The resultant LIFTed pixels, shown in Fig. 1(b), have the architectures: ITO/PEDOT:PSS/PVK/Alq $/$ /TBA)/Al. The pixel area is $\sim 0.6 \times 0.5 \mathrm{~mm}\left(0.3 \mathrm{~mm}^{2}\right)$, but smaller would be possible. $^{11}$

\section{Device characterization}

The LIFTed pixels were kept in an inert nitrogen environment, and contacted using a specially designed sample holder with contacting pins. To line up the cathode contacts with the contacting pins, silver was evaporated onto the samples, helped with silver paste where they may have been misaligned.

A Keithley 2400 sourcemeter was used to apply a bias across the OLEDs, as shown in Fig. 1(b), and measure the I-V characteristics. A Minolta LS-110 light-meter was linked 


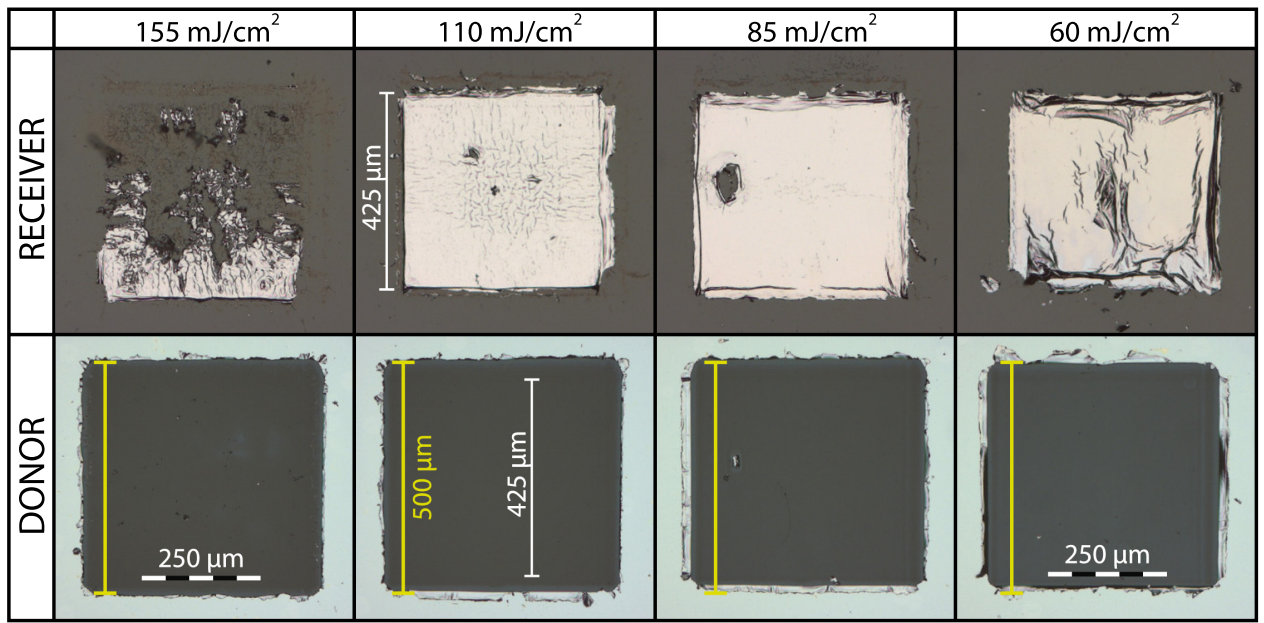

FIG. 3. $\mathrm{Alq}_{3} / \mathrm{Al}$ pixels deposited on a glass/PEDOT:PSS/PVK receiver substrate. The top images are the receiver substrate pixels, and the bottom images are the ablation crater on the donor substrate corresponding to the pixel above. Pixels deposited at four different laser fluences are shown.

to the sourcemeter by a home-built LABVIEW programme to allow synchronous luminance measurements, and the process has been outlined in detail in a previous article. ${ }^{6}$ Electroluminescence (EL) spectra were obtained using a Jobin Yvon Horiba FL-311 Fluorolog, profilometry measurements were taken using an Ambios XP-1 profilometer, and the pixels were looked at through standard light microscopes (Zeiss Axiovert and Stemmi).

\section{FABRICATION OF Alq ${ }_{3}$ PIXELS}

\section{A. $\mathrm{Alq}_{3}$ pixel fabrication results}

$90 \mathrm{~nm} \mathrm{Al} / 80 \mathrm{~nm} \mathrm{Alq}_{3}$ stacks have been transferred in the way shown in Fig. 1, and the fluence dependence of the pixels deposited by the LIFT process is shown in micrographs in Fig. 3. The $\mathrm{Alq}_{3}$ pixels can be deposited at a range of fluences as wide as that observed for polymeric OLEDs in previous research. ${ }^{11,12}$ This is remarkable for two reasons: first, $\mathrm{Alq}_{3}$ is a small molecule, meaning that the films are likely to have less internal cohesion than polymers, making them more brittle and susceptible to fragmentation; and second, $\mathrm{Alq}_{3}$ typically sublimes at fairly low temperatures, around $300{ }^{\circ} \mathrm{C} .{ }^{17}$ The fact that both of these effects are mitigated suggests that the organic layer is protected from both large thermal and mechanical loads.

The best pixel morphology in Fig. 3 came from transfer at a fluence of $85 \mathrm{~mJ} / \mathrm{cm}^{2}$. This fluence was used to fabricate functional pixels, shown from both above (a) and through the substrate (b) in Fig. 4. The quality of these $\mathrm{Alq}_{3}$ pixels, particularly when compared to the attempted LIFT of $\mathrm{Alq}_{3}$ pixels at atmospheric pressure, ${ }^{10}$ demonstrates the significant improvement in transfer that the following factors have: (1) the removal of air resistance by reducing the pressure, and (to a lesser extent) (2) the introduction of a gap.

Some length bars have been added to Fig. 3 to highlight how the pixel size differs with LIFT. For $110 \mathrm{~mJ} / \mathrm{cm}^{2}$, a white bar is drawn, measuring $425 \mu \mathrm{m}$. This highlights how transferring a pixel across a $15 \mu \mathrm{m}$ gap significantly reduces the pixel size by folding/crumpling/ripping at the edges (see a previous article by Shaw-Stewart et ll $^{10}{ }^{10}$. A second aspect shown by the yellow bar, of $500 \mu \mathrm{m}$, is how higher fluences create marginally larger ablation craters.
Fig. 4 shows the same pixel from above, onto the aluminium (a), and from below, through the glass, ITO, HTLs, and $\mathrm{Alq}_{3}$ (b). This not only shows the small cracks in more detail than Figs. 3 and 5 but also shows how defects may be visible from above that are less obvious through the substrate and, more importantly, vice versa. Examples of defects more obvious at the $\mathrm{Alq}_{3} / \mathrm{Al}$ interface than at the $\mathrm{Al} /$ air interface are shown circled in red. The pixel in Fig. 4 and other $\mathrm{Alq}_{3}$ devices have had their device characteristics tested in Sec. IV.

A direct comparison between $\mathrm{Alq}_{3}$ and polymeric PFN pixels (from an earlier article by Shaw-Stewart et al. ${ }^{12}$ ), transferred at the same fluences with the same Al thickness is shown in Fig. 5. This confirms that the small molecule $\mathrm{Alq}_{3}$ pixels are more prone to morphological defects such as cracks and folds than the polymeric PFN pixels.

\section{B. $\mathrm{Alq}_{3}$ pixel fabrication discussion}

The observation that the $\mathrm{Alq}_{3}$ does not decompose at fluences below $150 \mathrm{~mJ} / \mathrm{cm}^{2}$ indicates that at the laser fluences less than $100 \mathrm{~mJ} / \mathrm{cm}^{2}$, used for the optimised transfers such as the pixel in Fig. 4, the temperature in the organic layer over the aluminium is certainly below $300{ }^{\circ} \mathrm{C}$. ${ }^{17}$ Despite the fact that this is probably higher than the decomposition

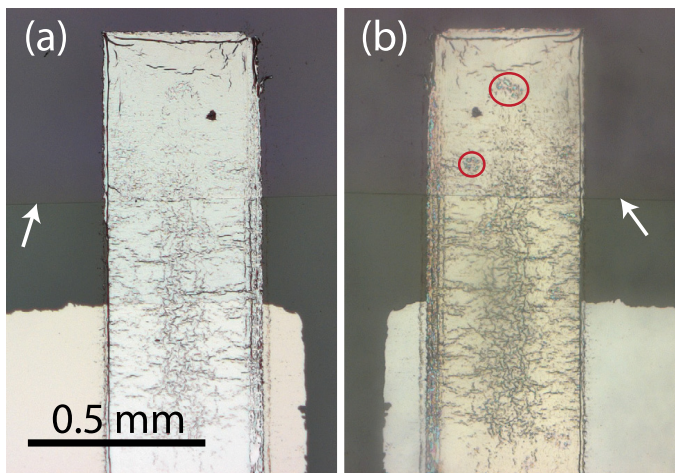

FIG. 4. A transferred $\mathrm{Alq}_{3} / \mathrm{TBA} / \mathrm{Al}$ pixel looking from above the deposition onto the aluminium (a), and through the glass substrate to the $\mathrm{Alq}_{3} / \mathrm{PVK}$ interface (b). The ITO is only coated on the glass substrate in the top half of the images only, above the line highlighted by the arrows. The pixel was transferred at a laser fluence of $85 \mathrm{~mJ} / \mathrm{cm}^{2}$. 


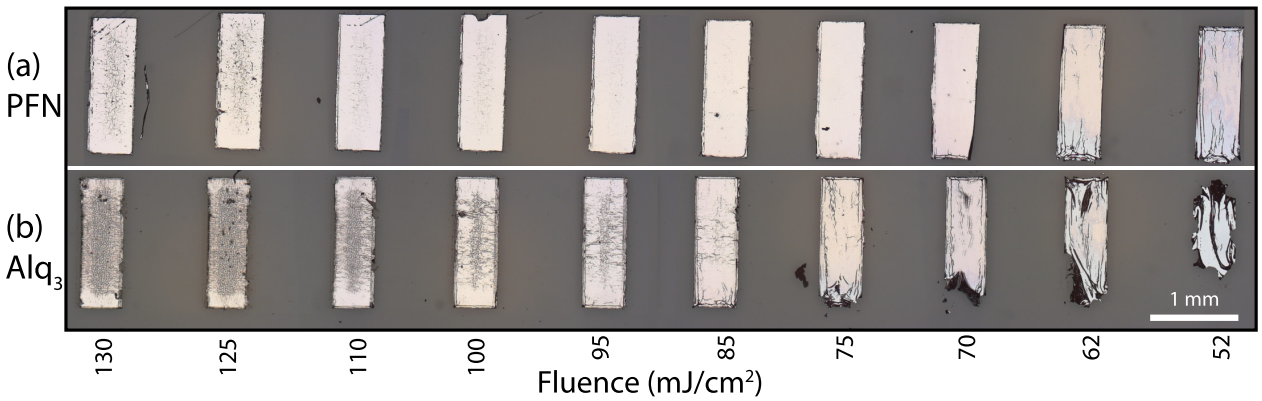

FIG. 5. A comparison between (a) an alcohol-soluble polyfluorene (PFN, from an earlier study ${ }^{12}$ ) and (b) $\mathrm{Alq}_{3}$ pixels transferred in identical conditions, with the only difference the organic layer (50 $\mathrm{nm} \mathrm{PFN} \mathrm{vs} 80 \mathrm{~nm} \mathrm{Alq}_{3}$. temperature of the polymeric materials investigated (MEH$\mathrm{PPV}, \mathrm{PFO}$, and $\mathrm{PFN}$ ), any onset of $\mathrm{Alq}_{3}$ sublimation is likely to have a greater effect upon pixel morphology than polymeric decomposition, due to the faster kinetics of sublimation, and this is not observed. However, there are fine cracks or folds which are particularly obvious when compared with PFN in Fig. 5.

The fine cracks and folds in the $\mathrm{Alq}_{3}$ in Figs. 3-5 are similar to those obtained when LIFT of $\mathrm{Alq}_{3}$ pixels at atmospheric pressure was attempted. ${ }^{10}$ Like the polymeric cracks obtained, for MEH-PPV 6 and PFO, ${ }^{10}$ the cracks are probably the result of the brittleness in the film, probably from mechanical stress in the transfer process. If the brittle fractures were from rapid heating and cooling, then it may be expected that the small molecule $\mathrm{Alq}_{3}$ would be less affected than the large molecules of polymers. Given that the polymers are typically more ductile in response to mechanical stress than the small molecule $\mathrm{Alq}_{3}$, the observation that $\mathrm{Alq}_{3}$ pixels have more cracks in it than the polymeric PFN in Fig. 5 backs up the hypothesis that the brittle fractures come from mechanical stress.

\section{IV. $\mathrm{Alq}_{3}$ small-molecule OLED (SMOLED) FUNCTIONALITY}

\section{A. Conventional $\mathrm{Alq}_{3}$ device results}

Due to the ubiquity of $\mathrm{Alq}_{3}$ in the OLED literature, it is easy to compare the devices to devices fabricated by other groups. The conventionally fabricated devices were made to act as a control for the LIFTed pixels, as there could be variation in material purity and film-forming apparatus, among other variables. Without a control, any responsibility for abnormal features of the LIFTed pixel device characteristics may be mistakenly attributed to the LIFT process.

Fig. 6(a) shows the external quantum efficiency (EQE) plotted against current density of the conventionally fabricated $\mathrm{PVK} / 80 \mathrm{~nm} \mathrm{Alq}_{3} / \mathrm{Al}$ device with the some EQEs reported from the literature for comparison. The device is better than older PVK/Alq ${ }_{3}$ devices from the $1990 \mathrm{~s} .{ }^{19,20}$ It is easy to speculate on differences, but one difference noted in the articles is that all the measurements from Jiang et al. ${ }^{19}$ and Berthelot et al. ${ }^{20}$ were undertaken in ambient conditions (unlike Tang and Van Slyke ${ }^{13}$ and Li et al., ${ }^{18}$ which were kept in inert oxygen-free atmospheres). It is particularly interesting that the EQE value from Jiang et al. ${ }^{19}$ is so close to the value obtained here $(0.25 \%$ vs $0.4 \%)$. The original OLED by Tang and Van Slyke ${ }^{13}$ has a good efficiency, partly not only because it was made under a very well controlled oxygen-free atmosphere but also because the charge-injection was optimised using a hole-transporting diamine and a low work-function $\mathrm{Mg}: \mathrm{Ag}$ alloyed cathode. Whilst a different hole-transporting layer may have helped a bit, the main limitation is probably the lower work function cathode helping electron-injection.

Fig. 6(b) shows the current density and luminance as a function of voltage. As can be seen, the luminance reaches very high brightness levels above $1000 \mathrm{~cd} / \mathrm{m}^{2}$ at relatively low current densities. The operating voltage is fairly high which is partly due to the high thickness of the $\mathrm{Alq}_{3}(80 \mathrm{~nm})$, and partly due to the charge injection at the electrodes which is probably not quite ideal. The $\mathrm{J}-\mathrm{V}$ and $\mathrm{L}-\mathrm{V}$ curves in Fig. 6(b) are in very good agreement with those in Ref. 19.

\section{B. LIFTed $\mathrm{Alq}_{3}$ pixel results}

The main aim of this study is to demonstrate the versatility of the LIFT process. After the fabrication of conjugated
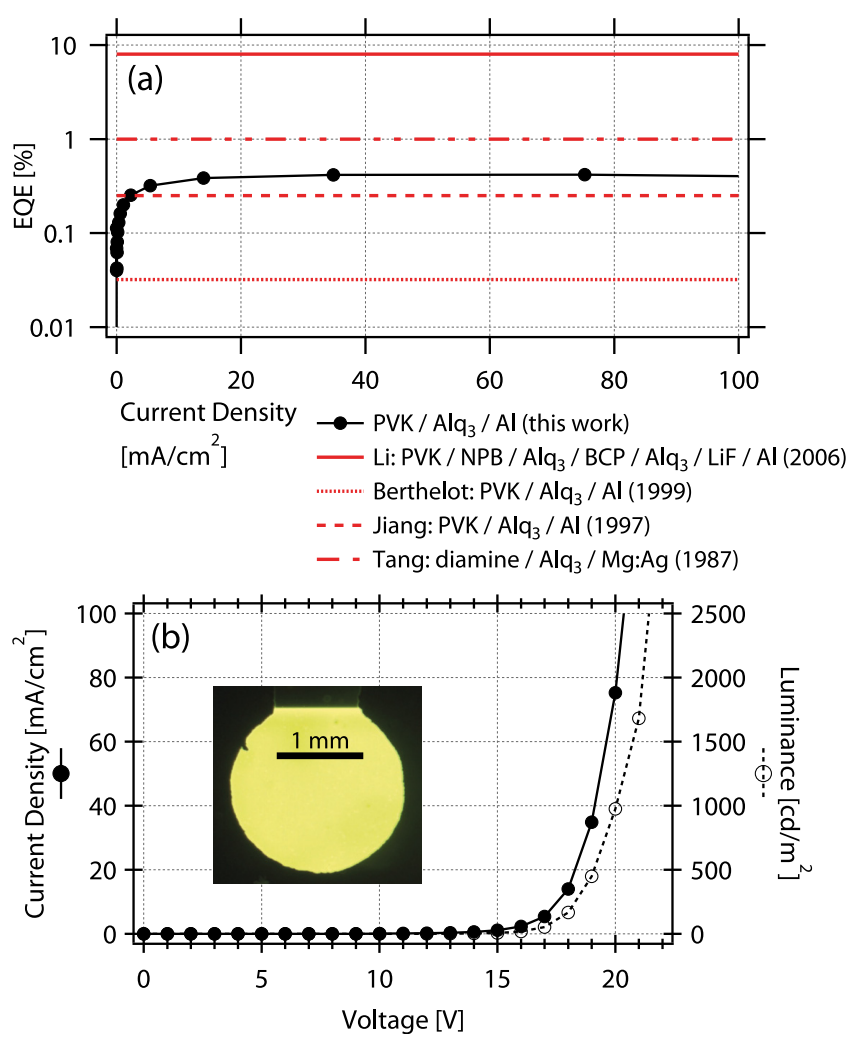

FIG. 6. (a) The EQE is plotted as a function of current density (J) for the conventionally fabricated $\mathrm{PVK} / \mathrm{Alq}_{3} / \mathrm{Al}$ device from this study compared with the best $\mathrm{Alq}_{3}$ devices with $\mathrm{PVK}$ and $\mathrm{Al},{ }^{18}$ earlier $\mathrm{PVK} / \mathrm{Alq}_{3} / \mathrm{Al}$ devices, ${ }^{19,20}$ and the original OLED with a diamine/Alq $3 / \mathrm{Mg}: \mathrm{Ag}$ structure. ${ }^{13}$ (b) The current density (J) and luminance (L) as a function of voltage (V) for the conventionally fabricated $\mathrm{PVK} / \mathrm{Alq}_{3}$ device from this study. 
polymer MEH-PPV OLED pixels, ${ }^{5,6}$ the research has continued to focus on polymeric materials; $\mathrm{PFO}^{11}$ and PFN. ${ }^{12}$ The fabrication of SMOLEDs by LIFT demonstrates that this variant of LIFT with a TP DRL is potentially applicable to all solid thin-film OLED materials.

In addition to just demonstrating a proof of principle, TBA was added onto the aluminium cathode to improve electron injection (as was done with the PFO in an earlier study by Shaw-Stewart et al. ${ }^{11}$ ). Fig. 7 shows a comparison of the conventional and LIFTed pixel device performances. Whilst the LIFTed pixels are clearly not quite as good as the conventional device, they appear to have a similar turn-on voltage in Fig. 7(b), and not too high operating voltages (they are comparable to the PFO and PFN devices). In terms of efficiency, in Fig. 7(a), the LIFTed pixels exhibit good external quantum efficiencies above $0.05 \%$.

When comparing the LIFTed pixels with and without TBA, the $\mathrm{Alq}_{3}$ devices show a significant difference. The pixel device characteristics in Fig. 7(b) show that the peak $\mathrm{EQE}$ increases from about $0.06 \%$ without TBA to $0.19 \%$ with TBA. This improvement with $\mathrm{Alq}_{3}$ is even more marked than for PFO in Shaw-Stewart et al. ${ }^{11}$ The operating voltage is also improved significantly in Fig. 7 with a reduction (at $\sim 50 \mathrm{~mA} / \mathrm{cm}^{2}$ ) from $31 \mathrm{~V}$ without TBA to $26 \mathrm{~V}$ with TBA. LIFTed pixels shown in Figs. 8(a)-8(c) show a pixel without TBA, and d-f show a pixel with TBA. The uniformity of the pixel electroluminescence in Figs. 8(b) and 8(e) demonstrates that there is no distinct difference in the pixel morphology with and without TBA.

The pixels before, during, and after operation is shown in Fig. 8, and demonstrate that the morphological defects mainly come from the transfer rather than operation. Whilst

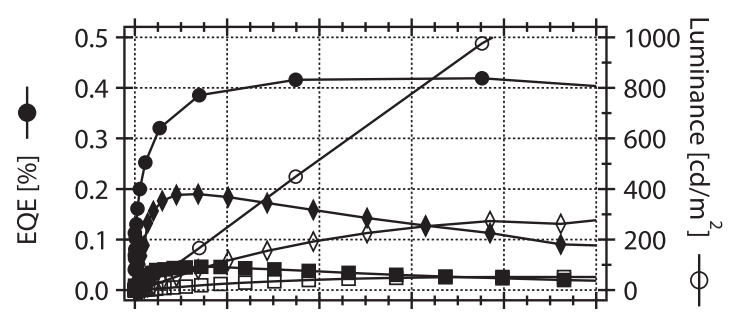
(a) 0
$\begin{array}{llll}20 & 40 & 60 & 80 \\ \text { Current Density } & {\left[\mathrm{mA} / \mathrm{cm}^{2}\right]}\end{array}$

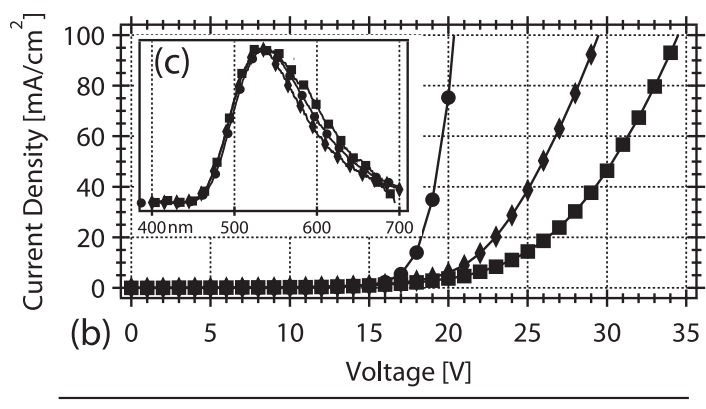

$\rightarrow$ LIFTed Al $\rightarrow$ LIFTed Al / TBA $\rightarrow$ Conventional Al

FIG. 7. A comparison of LIFTed pixel (square, just $\mathrm{Alq}_{3}$, and diamond, with TBA interlayer) and conventionally fabricated (circle) device characteristics. The graphs show the EQE and luminance as a function of current density at the top (a), current density as a function of voltage at the bottom (b), and the EL spectra as an inset (c). The LIFTed pixel characteristics used here are from pixels transferred at $85 \mathrm{~mJ} / \mathrm{cm}^{2}$.

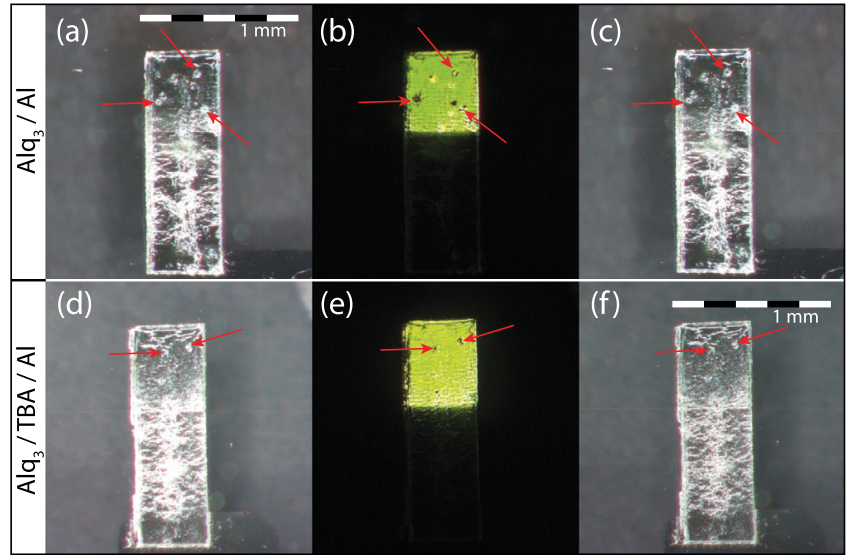

FIG. 8. Micrographs of an $\mathrm{Alq}_{3}$ pixel with an $\mathrm{Al}$ cathode before (a), during (b), and after (c) operation, and micrographs of $\mathrm{Alq}_{3} / \mathrm{TBA} / \mathrm{Al}$ pixel before (d), during (e), and after (f) operation. Both the pixels were transferred at a fluence of $85 \mathrm{~mJ} / \mathrm{cm}^{2}$.

this may appear to be a disadvantage with the LIFT process, the pixels do not exhibit significantly more defects than the polymers in previous studies, typified by the PFN pixels in Fig. 5(a), and show the potential to be optimised. In fact, the lack of black-spot defects in the OLEDs from operation due to oxygen or water contamination reflects positively upon LIFT. ${ }^{21} \mathrm{Alq}_{3}$ appears to transfer as well as any of the other materials with the optimised LIFT conditions of 1 mbar and a $15 \mu \mathrm{m}$ gap. The electroluminescence spectra all match very well, and the LIFTed pixel efficiencies are only just below the conventional device efficiencies, which perhaps perform better than polymers because the detrimental effects of aluminium evaporation on polymers are not so marked with $\mathrm{Alq}_{3}$.

Fig. 9 shows a final interesting feature of the data on LIFTed $\mathrm{Alq}_{3}$ pixels, where a slight trend in device performance was observed as a function of laser fluence. The lower the laser fluence of deposition, the higher luminance for a given current density/voltage (i.e., the higher the efficiency). Fig. 9 serves to show both this increase in efficiency at lower laser fluences, and to show the absolute values for luminance: over $300 \mathrm{~cd} / \mathrm{m}^{2}$. The insets also give some idea of the



FIG. 9. A device performance comparison of $\mathrm{Alq}_{3} / \mathrm{TBA} / \mathrm{Al}$ pixels transferred at different fluences: 90,85 , and $75 \mathrm{~mJ} / \mathrm{cm}^{2}$. Micrographs of the relevant pixel devices are shown as insets. 
pixel morphologies. All three pixels show some defects, like those in Fig. 8, but the lowest fluence, $75 \mathrm{~mJ} / \mathrm{cm}^{2}$, is clearly the pixel with the most defects. This does follow the pattern set by the polymers, particularly observed in the LIFT optimisation study. ${ }^{10}$

\section{Influence of TBA layer on LIFTed pixels}

For the LIFTed $\mathrm{Alq}_{3}$ pixels shown in Fig. 7, there is a distinct improvement in the device performance when the TBA layer is added onto the Al cathode. The explanation for this effect has been attributed to the formation of a dipolar layer creating a significant negative work function shift to the electrode surface. ${ }^{14}$ The LIFT process has allowed more investigation into the process by which the TBA aids electron injection, and a comparison of device characteristics of pixels made with two types of polyfluorenes, $\mathrm{PFO}^{11}$ and alcohol-soluble PFN, ${ }^{12}$ is shown in Table I.

As shown in Fig. 2, the pretext for using TBA was that it creates a dipolar layer on the cathode surface. The original hypothesis depended on the formation of a layer via some sort of reaction between the $\mathrm{OH}^{-}$with the surface, giving the dipolar structure of the negative hydroxide ion $\left(\mathrm{OH}^{-}\right)$ and the positive counterion $\left(\mathrm{N}_{(}\left(\mathrm{C}_{4} \mathrm{H}_{9}\right)_{4}{ }^{+}\right)$. The monolayer structure proved to be unlikely for TBA, despite the large reduction in the cathode work function. ${ }^{14}$

A comparison between the results of different materials with TBA in Table I could aid understanding of how TBA works. Comparing just the LIFTed devices first, only $\mathrm{Alq}_{3}$ exhibits a significant decrease in the operating voltage, although PFO appears to have a slight decrease. The presence of TBA significantly increases the EQE of $\mathrm{Alq}_{3}$ pixels by over $600 \%$, but only increases the PFO by $\sim 50 \%$ and even causes the PFN pixel EQE to decrease. Evidently, TBA has the most positive effect on $\mathrm{Alq}_{3}$. There are clearly numerous differences between the materials that could account for the bigger effect on $\mathrm{Alq}_{3}$. However, one simple explanation is the deposition method; $\mathrm{Alq}_{3}$ is evaporated and the polymers PFO and PFN are spin-coated. The spin-coating could remove

TABLE I. Device performances for various device architectures, at a current density (CD) of $\sim 40-50 \mathrm{~mA} / \mathrm{cm}^{2}$. The luminance (Lum), luminous efficiency (LE), and EQE are all shown for the $\mathrm{Alq}_{3}$ SMOLEDs fabricated in this article as well as other OLEDs using polymeric materials.

\begin{tabular}{lcccccl}
\hline \hline & & $\begin{array}{c}\text { Bias } \\
(\mathrm{V})\end{array}$ & $\begin{array}{c}\mathrm{CD} \\
\left(\mathrm{mJ} \mathrm{cm}^{-2}\right)\end{array}$ & $\begin{array}{c}\text { Lum } \\
\left(\mathrm{cd} \mathrm{m}^{-2}\right)\end{array}$ & $\begin{array}{c}\mathrm{LE} \\
\left(\mathrm{cd} \mathrm{A}^{-1}\right)\end{array}$ & $\begin{array}{c}\mathrm{EQE} \\
(\%)\end{array}$ \\
\hline $\mathrm{Alq}_{3}$ & LIFT Al & 30 & 46.3 & 44.4 & 0.096 & 0.01 \\
& LIFT Al/TBA & 25 & 38.7 & 192.3 & 0.50 & 0.062 \\
& Convent Al & 19 & 34.9 & 448.7 & 1.29 & 0.21 \\
$\mathrm{PFN}^{\mathrm{a}}$ & LIFT Al & 16 & 43 & 44.9 & 0.11 & 0.043 \\
& LIFT Al/TBA & 17 & 37.7 & 28.1 & 0.075 & 0.030 \\
& Convent Al & 17 & 41.6 & 0.3 & 0.0007 & 0.0003 \\
PFO $^{\mathrm{b}}$ & LIFT Al & 22 & 51 & 306.2 & 0.60 & 0.27 \\
& LIFT Al/TBA & 21 & 48.32 & 540 & 0.89 & 0.40 \\
& Convent Al & 18 & 51.16 & 25.4 & 0.05 & 0.042 \\
& Convent Ca & 13 & 49.23 & 102.3 & 0.21 & 0.17 \\
\hline \hline
\end{tabular}

${ }^{\text {a }}$ Partly in Shaw-Stewart et al. ${ }^{12}$

${ }^{\mathrm{b}}$ From Shaw-Stewart et al. ${ }^{11}$ some of the layer. PFN is spin-coated from a methanol solution, ${ }^{12}$ like the TBA, meaning that it may be able to redissolve a substantial amount of the TBA layer. In addition, the TBA could also hinder performance if it acts as a trap within the PFN layer. PFO is spin-coated from a toluene:xylene solution, still types of alcohol. This would be less likely to redissolve the TBA layer than methanol, but may still damage the layer significantly enough to reduce the improvement. However, it must be stressed that there could be other explanations based on the deposition process, or indeed based on other material differences between $\mathrm{Alq}_{3}, \mathrm{PFN}$, and PFO.

\section{Discussion of $\mathrm{Alq}_{3}$ SMOLED performances}

The operation of LIFTed $\mathrm{Alq}_{3}$ pixels demonstrates that LIFT may be applied to all types of OLED materials successfully. The LIFTed $\mathrm{Alq}_{3}$ pixels exhibit efficiencies of about the same order of magnitude as the conventional devices, and generally show good device performance. In previous comparative studies using polymeric light-emitting layers, ${ }^{6,11,12}$ the LIFTed pixels have nearly always exhibited improved performance relative to the conventional devices. The main reason given for the improved performance of LIFTed devices was that aluminium evaporation onto the polymeric materials damaged the device performance. ${ }^{22}$ It is likely that although aluminium will still react with the $\mathrm{Alq}_{3}$ when fabricating conventional devices, ${ }^{23}$ the consequence is not bad, and may possibly be positive for electron injection, and overall device performance.

TBA significantly improved the LIFTed pixel performances, and one proposed mechanism for the electron injection of TBA is well explained in Fig. 2; the $\mathrm{OH}^{-}$reacts with acidic sites on the aluminium (assuming it is partly oxidised) to create negatively charged surfaces which will encourage the large positive counterions $\mathrm{N}\left(\mathrm{C}_{4} \mathrm{H}_{9}\right)_{4}{ }^{+}$to assemble as shown in Fig. 2. ${ }^{14,15}$ This creates a dipolar layer which has an internal electric field, effectively driving electrons through from the $\mathrm{Al}$ cathode improving electron injection (e.g., Ref. 24). However, unlike the original hypothesis, ${ }^{15}$ the TBA may not be forming a monolayer (in fact, it is very unlikely to be doing so), but it may form a thicker film which can still create a strong dipole, both at the Al/TBA interface, and within the TBA film. ${ }^{24}$ Nevertheless, this is not incompatible than the original idea that the adsorption starts with a weak acid-base reaction. The idea of thicker than few-layer TBA supports the hypothesis that spin-coating a polymeric layer on top of the TBA will (partially) dissolve the TBA layer, outlined in Sec. IV C.

Another aspect of the $\mathrm{Alq}_{3}$ pixels which is worth looking at in detail is the morphology of the pixels. The comparison with the polymeric OLED pixels in Fig. 5 is particularly revealing as it highlights how many fewer defects the polymeric pixels have. Rather than different mechanical properties, already mentioned in Sec. III B, one other explanation for the morphological differences between $\mathrm{Alq}_{3}$ pixels and the polymeric material pixels comes from the sublimation temperature of $\mathrm{Alq}_{3}$. A detailed analysis of the $\mathrm{Alq}_{3}$ bought from Aldrich has shown an onset of sublimation at $300^{\circ} \mathrm{C} .{ }^{17}$ Even though this is roughly the same as the thermal 
decomposition temperatures of standard light-emitting conjugated polymers, ${ }^{25}$ as a phase change rather than a thermal reaction (governed by Arrhenius kinetics), it is more likely to happen on the short time-scales of the laser pulse. An indication of the nature of pulse laser thermal degradation is indirectly shown via a recent study on the deposition of $\mathrm{MEH}-\mathrm{PPV}, \mathrm{PFO}$, and $\mathrm{Alq}_{3}$ by resonant infrared pulsed laser deposition (RIR-PLD) and resonant infrared matrix-assisted pulsed laser evaporation (RIR-MAPLE). ${ }^{26}$ Whilst $\mathrm{Alq}_{3}$ could be deposited via RIR-PLD without significant damage to the material, this was not the case for the polymeric materials, which were all better suited to the "softer" RIR-MAPLE technique where the polymer is dissolved in a host matrix at a concentration of $\sim 1 \%$. This shows how the $\mathrm{Alq}_{3}$ sublimes without any chemical structure damage, but the polymers will not sublime, or even melt without some chemical structure damage. This suggests that any effects of $\mathrm{Alq}_{3}$ sublimation on the LIFT pixels could manifest themselves as morphological problems from small areas subliming.

This hypothesis is partly backed up by the data in Fig. 9, particularly by the low $90 \mathrm{~mJ} / \mathrm{cm}^{2}$ vs $75 \mathrm{~mJ} / \mathrm{cm}^{2}$ device performance data, where the lower fluence pixel is considerably more efficient than the higher fluence pixel. However, the images tell a different story-the lower the fluence the more heterogeneity and defects in the pixel. This suggests that although there may be small levels of sublimation damage to the $\mathrm{Alq}_{3}$ film, the main source of morphological defects is probably due to mechanical load.

\section{CONCLUSIONS}

Laser-induced forward transfer has been used to fabricate functional small molecule OLED pixels by transferring a stack of the cathode ( $\mathrm{Al})$ and the small molecule lightemitting layer $\left(\mathrm{Alq}_{3}\right)$ across a $15 \mu \mathrm{m}$ gap onto a pre-patterned anode receiver substrate. $\mathrm{Alq}_{3}$ devices have been fabricated in a conventional fabrication process as control devices to the LIFTed pixels.

Peak LIFTed $\mathrm{Alq}_{3}$ pixel efficiencies of $0.04 \%$ of the conventionally fabricated devices were obtained, and this was improved to almost $0.2 \%$ with the addition of a TBA electron-injecting layer between the $\mathrm{Al}$ and $\mathrm{Alq}_{3}$ films. The evaporation of the $\mathrm{Alq}_{3}$ layer after the deposition of the TBA gives new evidence that the TBA forms a continuous dipolar layer (rather than mixing with the light-emitting layer), unobtainable for solution-processed polymeric films. Whilst the $\mathrm{Alq}_{3}$ /TBA LIFTed pixel performance is good, it is still less than half as efficient as the conventionally fabricated devices.

The morphology of the LIFTed pixels has been investigated in detail. More fine cracks and folding were observed for small molecule $\mathrm{Alq}_{3}$ when compared to a polymeric layer. Whilst not ideal, these defects, probably of mechanical origin, suggest that the thermal load is less significant than the mechanical load. There is definitely still room for optimising the full workflow so that the scale of the defects can be mini- mised, but the extent to which this would improve the pixel morphology is unclear without further work.

The successful deposition of $\mathrm{Alq}_{3}$ pixels marks an important step forward for the LIFT process, proving that even brittle materials can be successfully transferred when the LIFT process is optimised by mechanisms such as reducing the atmospheric pressure, and controlling the donor-receiver gap. It demonstrates that the variant of LIFT presented here is a particularly versatile thin-film pixel deposition technique.

\section{ACKNOWLEDGMENTS}

The authors would like to thank Ylenia Maniglio (Empa) for synthesizing the triazene polymer. Financial support is gratefully acknowledged from the FP7 European project e-LIFT (Project No. 247868-call FP7-ICT-2009-4) and the Swiss National Science Foundation.

${ }^{1}$ R. Braudy, Proc. IEEE 57, 1771 (1969).

${ }^{2}$ J. Bohandy, B. Kim, and F. Adrian, J. Appl. Phys. 60, 1538 (1986).

${ }^{3}$ C. Arnold, P. Serra, and A. Piqué, MRS Bull. 32, 23 (2007).

${ }^{4}$ P. Semenza, Inf. Display 27, 14 (2011). http://www.informationdisplay.org/ article.cfm? year $=2011 \&$ issue $=10 \&$ file $=$ art 4 .

${ }^{5}$ R. Fardel, M. Nagel, F. Nüesch, T. Lippert, and A. Wokaun, Appl. Phys. Lett. 91, 061103 (2007).

${ }^{6}$ J. Shaw-Stewart, T. Lippert, M. Nagel, F. Nüesch, and A. Wokaun, ACS Appl. Mater. Interfaces 3, 309 (2011).

${ }^{7}$ N. Kattamis, N. McDaniel, S. Bernhard, and C. Arnold, Org. Electron. 12, 1152 (2011).

${ }^{8}$ M. Wolk, S. Lamansky, and W. Tolbert, SID Int. Symp. Digest Tech. Papers 39, 511 (2008).

${ }^{9}$ N. Kattamis, N. McDaniel, S. Bernhard, and C. Arnold, Appl. Phys. Lett. 94, 103306 (2009).

${ }^{10}$ J. Shaw-Stewart, B. Chu, T. Lippert, Y. Maniglio, M. Nagel, F. Nüesch, and A. Wokaun, Appl. Phys. A, Mater. Sci. Process. 105, 713 (2011).

${ }^{11}$ J. Shaw-Stewart, T. Lippert, M. Nagel, F. Nuesch, and A. Wokaun, Appl. Phys. Lett. 100, 203303 (2012).

${ }^{12}$ J. Shaw-Stewart, T. Lippert, M. Nagel, F. Nüesch, and A. Wokaun, ACS Appl. Mater. Interfaces 4, 3535 (2012).

${ }^{13}$ C. Tang and S. Van Slyke, Appl. Phys. Lett. 51, 913 (1987).

${ }^{14}$ F. Nüesch, L. Rothberg, E. Forsythe, Q. Toan Le, and Y. Gao, Appl. Phys. Lett. 74, 880 (1999).

${ }^{15}$ F. Nüesch, Y. Li, and L. Rothberg, Appl. Phys. Lett. 75, 1799 (1999).

${ }^{16}$ J. Stebani, O. Nuyken, T. Lippert, and A. Wokaun, Makromol. Chem., Rapid Commun. 14, 365 (1993).

${ }^{17}$ T. Lee and M. Lin, Cryst. Growth Des. 7, 1803 (2007).

${ }^{18}$ W. Li, R. Jones, S. Allen, J. Heikenfeld, and A. Steckl, J. Display Technol. 2, 143 (2006).

${ }^{19}$ X. Jiang, Y. Liu, X. Song, and D. Zhu, Synth. Met. 87, 175 (1997).

${ }^{20}$ L. Berthelot, J. Tardy, M. Garrigues, P. Cremillieu, J. Joseph, and B. Masenelli, Opt. Mater. 12, 261 (1999).

${ }^{21}$ M. Schaer, F. Nüesch, D. Berner, W. Leo, and L. Zuppiroli, Adv. Funct. Mater. 11, 116 (2001).

${ }^{22}$ K. Demirkan, A. Mathew, C. Weiland, M. Reid, and R. Opila, J. Appl. Phys. 103, 034505 (2008).

${ }^{23}$ T. Nguyen, J. Ip, P. Jolinat, and P. Destruel, Appl. Surf. Sci. 172, 75 (2001).

${ }^{24}$ Y. Zhou, C. Fuentes-Hernandez, J. Shim, J. Meyer, A. Giordano, H. Li, P. Winget, T. Papadopoulos, H. Cheun, J. Kim, M. Fenoll, A. Dindar, W. Haske, E. Najafabadi, T. Khan, H. Sojoudi, S. Barlow, S. Graham, J.-L. Brédas, S. Marder, A. Kahn, and B. Kippelen, Science 336, 327 (2012).

${ }^{25}$ S. Xiao, M. Nguyen, X. Gong, Y. Cao, H. Wu, D. Moses, and A. Heeger, Adv. Funct. Mater. 13, 25 (2003).

${ }^{26}$ H. Park, K. Schriver, and R. Haglund, Appl. Phys. A, Mater. Sci. Process. 105, 583 (2011). 